\title{
Conceptual model for an ICT-enabled educational platform for collaborative design
}

\author{
B. Şenyapilı \\ Department of Architecture, Faculty of Art, Design and Architecture, Bilkent University, Ankara, Turkey \\ C.J. Anumba \\ Department of Architectural Engineering, The Pennsylvania State University, State College, USA
}

\begin{abstract}
Almost all design related practices (architecture, architectural engineering, interior architecture/design, landscape architecture, urban design, industrial design, fashion and visual communication design) heavily depend on collaboration between colleagues from the same or different disciplines. However, practitioners who collaborate in practice, seldom have the opportunity to collaborate during design education. Having the opportunity to collaborate on educational design projects and acquiring collaboration skills and experience during design education should contribute to the quality and efficiency of future professional collaborations. Recent advancements in information and communication technologies have enabled collaboration during the process of the design studio, especially between geographically dispersed parties, but initiating and establishing such collaboration opportunities depends on the parties' singular efforts. This paper reviews previous and ongoing efforts at introducing multi-disciplinary collaborative design studios in Turkey and the USA and proposes a conceptual model for an online collaboration network that aims at promoting and facilitating collaboration among design schools and design instructors/students/ researchers. The network, establishing an educational platform for collaborative design (EPCOD) is intended to comprise a digital pool of design projects, enabling potentials of design collaboration for students from the same or different disciplines. It is designed to be accessible to all design schools all over the world, forming a specific and rich platform for design education. The role of current and emerging information and communication technologies in facilitating the proposed network-based collaboration is also discussed.
\end{abstract}

\section{INTRODUCTION}

The emergence of information and communication technologies (ICT) has significantly affected design practices and education in a relatively short time interval (Wang, 2012; Pentilla, 2006). The positive outcomes of the utilization of ICT platforms in design education (Kalay 2004; Yeung et al 1998), the necessity of integrating new technologies into design education (Oxman, 2007), and the contribution of Internetbased systems to design education (Nguyen and Bodi, 2007; Sigle et al., 2005; Koohang and Durante, 2003; Simoff and Maher, 1997) are stated in previous studies. Another set of studies focus on how ICT facilitates and promotes design collaboration (Anumba et al., 2008; Fruchter et al. 2007; Shelbourn et al. 2004; McCormick, 2004; Everitt et al., 2003; Blaszczyszyn, 2002; Cheng and Khan, 2000).

Design practices heavily depend on collaboration between colleagues from the same or different disciplines. Thus, acquiring collaboration skills and experience during design education is beneficial for the future practice of the disciplines. In today's design practice, where the boundaries of coverage between the different design disciplines are blurred, collaboration between different design related disciplines is proving to be essential in design education (Karakaya and Senyapili, 2008). According to Karakaya and Senyapili's study, this type of collaboration aids the students in recognizing each other's goals, perspectives, responsibilities and capabilities. It also allows for rehearsing what the professional work sharing would be like in terms of roles, responsibilities undertaken, ownership and leadership.

The Internet is now well established as an effective medium for information collection and dissemination almost in every field. Design is no exception. The opportunities for collaboration between different disciplines during design education are often limited by accreditation and other requirements, as well as by the limited time available in the curriculum. It can be argued that there is scope to address this by seeking to leverage the facilities offered by the Internet, which has not been used to its full capacity in this domain. Within this context, we propose a reference model, called EPCOD, aiming at utilizing the Internet as a medium for providing and/or facilitating design collaboration during design education. 
In this paper we first set forth the aim and scope of EPCOD and then we introduce the system structure and aspects of implementations. We discuss how the proposed model differs from similar models of collaboration. We elaborate on the potential benefits and challenges. Finally, we conclude by discussing how the model should be realized and what further studies may be established.

\section{PREVIOUS WORK}

\subsection{Collaborative design studies at Bilkent University}

The necessity of collaborative design is acknowledged for a long time at Bilkent University. The multidisciplinary structure of design practice asks for graduates who are equipped with collaboration skills. In the studies utilizing web-based collaborative learning (WBCL), administered by Bilkent University Faculty of Art, Architecture and Design (FADA) and Delft Technical University, it is found out that in order to foresee and resolve the problems that may occur in real life collaboration, it is beneficial for students to rehearse the process during design education (Karakaya and Senyapili, 2007). Therefore, at Bilkent University FADA, not only the curriculum is enhanced with collaborative courses that bring students of different departments together, but opportunities that involve participation of geographically dispersed groups are sought. The expectation is to be able to bring students of different disciplines/schools/ geographies to collaborate.

\subsection{Collaborative opportunities at the Pennsylvania State University}

At the Pennsylvania State University, collaborative design has been a feature of the architectural engineering program for many years. Recent advances in information and communication technologies have enabled new facets of multi-disciplinary collaboration. For example, the use of Building Information Modelling (BIM) is now becoming widespread within the AEC (Architecture, Engineering and Construction) sector of the industry. In parallel, the construction industry has been moving towards more collaborative modes of procurement, such as Integrated Project Delivery (IPD). To ensure that students are well equipped to function effectively within the emerging project environment, a new integrated design studio was developed. This was designed to include students from the four architectural engineering sub-disciplines (construction engineering, structural engineering, mechanical engineering and lighting/electrical), and from architecture and landscape architecture. This is considered the first six-discipline studio anywhere in the world. Groups of six students including one from each of the above disciplines were required to work on a collaborative design project based on IPD concepts and to use BIM as the platform for integration. The project brief was usually provided by a local client or consultant, and the students were tasked with developing a design taking into account a number of critical considerations. It was found that students were able to work effectively as a team once they established the roles of individual team members and assigned responsibilities. They found BIM a good platform for collaboration but often had to figure out how best to transfer information between the BIM model and specialist software used for various analyses. Interviews with the students at the end of the design project revealed that the students learned more by collaborating with the other disciplines than they would have learnt if they worked on their own. At the end of the exercise, the students had to present their designs to a jury made up of faculty and industry practitioners. For the exercise, the student teams were collocated and worked closely face-to-face, with minimal reliance on electronic communications. A natural extension of this study would be to explore how the student team collaboration would work when the team members are geographically distributed and need to use the BIM model as a collaboration tool.

\section{PROPOSED MODEL}

EPCOD stands for Educational Platform for Collaborative Design. It aims at introducing design schools with opportunities for sharing educational design projects and enabling scholars/instructors from different schools to collaborate. EPCOD is an Internet based network for meetings/discussion/sharing for design instructors/scholars/researchers. Design projects that are input by different design schools will constitute a digital project pool. This pool will enable parties from different design schools to be informed about what the others are doing; work on the same project simultaneously in collaboration or non-simultaneously, with the opportunity to discuss the outcomes later. EPCOD aims at weaving a collaboration network of different design schools and their members through a database. The database shall enable users to obtain information on the design schools looking for partners in a design project and/or post notices searching for partners. EPCOD is planned to be a specific network bringing design schools together. EPCOD is being developed to fulfill the following:

1) To form a network that connects geographically dispersed design schools, allowing them to input design projects and search for them.

Description: The digital project pool, which will be formed as users input data, will serve as an archive.

Sample use: A(ny) user may access the network

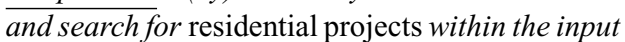
data. She may have a look at which parties collaborated on which domestic projects, specifications and results of the project. 
2) To promote/enable/facilitate collaboration between scholars from different design schools and/or professions.

Description: Instructors seeking for design collaboration partners from different schools and/or professions may use EPCOD to see available/ willing partners. Likewise, scholars who would like to participate in a project held by different parties may seek opportunities through EPCOD.

Sample use: A professor of architecture:

- may post a call for 3 rd year interior architecture students to collaborate on a 4 week restaurant design project with 20 architecture students.

- may look up to see whether there are any calls from urban design schools seeking 3rd year architectural students as partners on a project.

3) To establish a platform which students and scholars from different design schools and different professions can collaborate synchronously or asynchronously with colleagues and designers in other design schools.

Description: Scholars may use EPCOD to be informed about each other, get engaged in discussions/forums, exchange ideas, and collaborate on projects.

Sample use: A scholar may ask a question online for other students and professors to comment on. He/She may also work collaboratively with others at another location using the platform.

Within this framework, there are three components essential for the operation of the system:

Users. There needs to be at least two separate groups of students willing to collaborate. They may be separated in terms of geography and/or design discipline and/or the school they are affiliated with.

Process. The system may be utilized for one of the main operations below:

- input collaboration call

- search for a collaboration call

- upload details of concluded projects

- general searches (projects, collaborating partners, etc.)

Each call needs to be described within the determined id (Figure 1).

Each search may be operated with one or more parameters of the call id. The intersecting regions of the call ids and search parameters establish the potential for collaboration (Figure 2).

Parties may upload presentations of the concluded projects to contribute to the digital design pool.

Design space. Each collaborative design activity requires a physical or virtual design space. EPCOD does not provide a design space for the users. However, it allows the users to specify the choice of design space: Virtual Design Studios (VDS) or Collaborative Design Studios (CDS) for facilitating the meeting of common objectives.

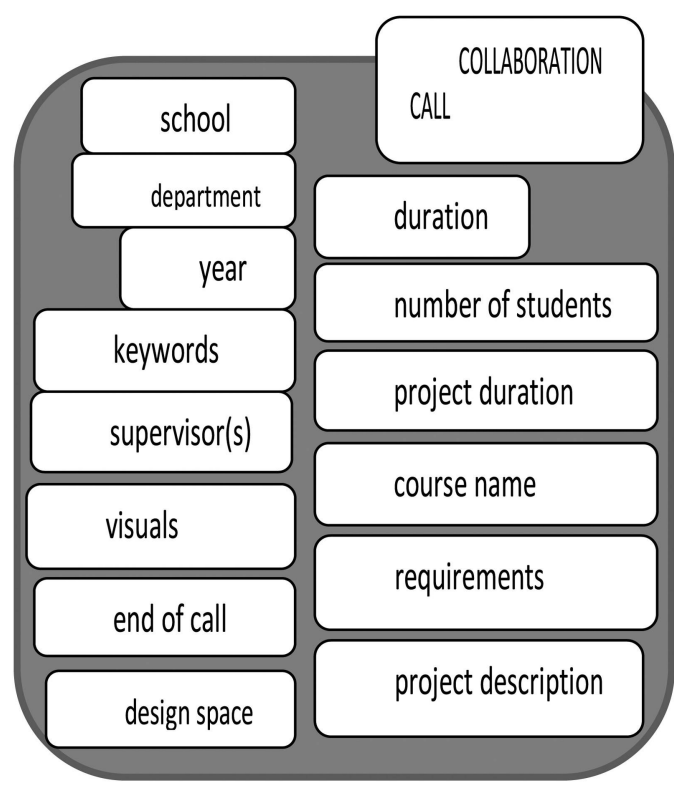

Figure 1. Sample collaboration call id.

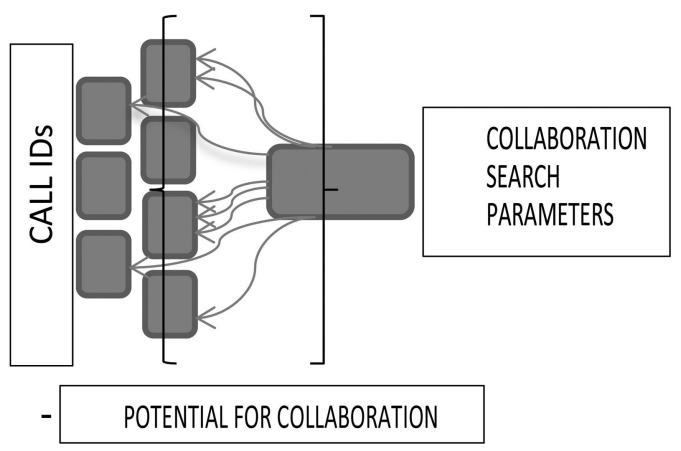

Figure 2. Potential for collaboration based on the call ids and search parameters.

The system in built up in 4 consecutive stages; first, we establish the system map. We list the required nodes within the system structure and lay out a relational network map. This map determines the basic structure of the web site, indicating the main portals and web page hierarchy. At this stage, Website Preference Scale (WSPS) (Rosen and Purington, 2004) is adopted for structuring EPCOD. WSPS defines the attributes that determine people's preferences and sets forth the requirements for a successful website design (Table 1).

Then we implement a pilot model based on the map obtained in the former phase. The pilot model comprises one or more branches of the overall system structure, the branch(es) demonstrating the most ramified part(s) of the system. At this phase, a limited user assessment may be carried out in order to test the efficiency and user-friendliness of the model. The phase will be terminated by a thorough assessment 
Table 1. The WSPS and website features (Rosen and Purinton, 2004, p. 792).

\begin{tabular}{lll}
\hline Coherence & Complexity & Legibility \\
\hline - Simplicity & - Variety in content (text and graphics) & - Mini home page on every \\
- Easy to read & - Changing graphics & subsequent page \\
- Use of categories & - Different categories of text & - Same menu on every page \\
- Absence of information overload & & - Site map \\
- Adequate font size & & \\
- Uncrowded presentation & & \\
\hline
\end{tabular}

of the model, with revisions to the model, if deemed necessary.

In the following phase, the actual system will be implemented. The web pages in the system map will each be designed and constructed separately. At the end of the phase the individual pages will be linked as determined in the system map. Simultaneously, the database structure of the system will be developed and implemented. User tests will be carried out in order to evaluate the system.

In the final phase, the system will be published on the Web. Pilot user assessments will be carried out, leading to system refinement. Brief descriptions of the site and an invitation will then be sent to a number of design schools.

\section{DISCUSSION}

The proposed EPCOD model is based on the fact that in professional life designs are often realized through the team work of several members of the same or different disciplines, who seldom had the opportunity to rehearse such collaborative skills during their education. For instance, professionally a housing project is realized by the contribution of architects, interior designers and landscape architects whereas in design education a student from one of those disciplines would have performed all the tasks, or assumed that some tasks are taken care of by the others. This paper argues that if the students have the chance to collaborate with others during education that would equip them with the skills of working in a team as well as collaborating with members of different disciplines. As such, they would know, before they went out in the field, the dynamics of a design team and the responsibilities undertaken by other disciplines. EPCOD aims at bringing students together during education. As such, architecture students from different geographies may collaborate on the same project going to the experience of sharing workloads, listening to and respecting each other's ideas, as well as developing ways to debate. Similarly, architecture students may collaborate with urban designers operating on the macro scale decisions made by the urbanists. While EPCOD provides a platform for providing collaboration opportunities, it also acts as an archive for storing the outcomes of these educational projects. The digital pool of projects and the digital archive formed by EPCOD are expected to serve the whole design community.

There are similar efforts in the design area:

* ArchNET (archnet.org) is a network supported by the Aga Khan Foundation and Massachusetts Institute of Technology (MIT) which provides a service to architects, urban planners, landscape architects with a special focus on Islamic art and architecture. It is an excellent platform for designers to get connected but it is not as specific as EPCOD is for collaboration purposes.

* OIKODOMOS (www.oikodomos.org) is a network supported by the European Union and is implemented by 7 schools in Europe focusing on housing projects. In comparison, EPCOD does not have specific project topics that would limit the number of users.

* Networks such as archINFORM (archinform.net) and ARCHIPLANET (www.archiplanet.org) provide a digital pool of projects similar to the proposed project. However, this archive covers existing buildings rather than a pool of educational projects as EPCOD proposes.

The sample model for EPCOD will be the eSTUDIO (www.engr.psu.edu/ae/thesis/about.htm) a platform developed by the Department of Architectural Engineering of Penn State University. The platform acts as an online repository of student projects and enables undergraduate senior students to find outside sponsors/collaborators for their capstone projects. The idea of e-STUDIO that aims at collaborating with professionals during education is transformed into the collaboration of students and schools in EPCOD.

Through the utilization of EPCOD, we anticipate that:

1) EPCOD will enable the design education community to be connected at all times, independent of temporal and geographical constraints; following the trends/ developments in design education worldwide;

2) EPCOD shall especially create and facilitate collaboration between different design schools and students, equipping them with collaboration skills during education; 
3) The digital pool of design projects shall contribute to the information flow between design schools, enabling them to be informed about the projects being undertaken in design education around the world, and enabling them to participate in collaborative projects.

4) The system model of EPCOD may later be adapted to other professional fields and their education.

We do not anticipate problems of major magnitude in the conceptual and specific modeling phases. However, in implementation modeling phase there may be difficulties in user's adaptation to the system. Moreover, the maintenance of the system needs to be carefully managed to ensure the sustainability of the system in the future.

\section{CONCLUSIONS}

Several design schools are already providing opportunities for students from different disciplines to collaborate on projects. In many cases, these students are collocated while in others, they are distributed and rely on the internet to facilitate collaboration. At Penn State University, students from six disciplines (architecture, landscape architecture, construction engineering and management, structural engineering, mechanical engineering, and lighting/electrical engineering) collaborate in a BIM-based design studio. There are numerous examples of Web-based bilateral or multi-lateral design collaborations that bring students together. The EPCOD platform proposed here seeks to build on these collaborations by adding a searchable repository of design school projects and a networking facility that enables schools that are not part of established collaborative projects to find partners and/or see what projects others are working on. Appropriate protocols will need to be established to ensure that intellectual property, and other appropriate protections are observed by participants, especially when sensitive projects are involved. However, it is expected that EPCOD will enable free-flowing exchange of information between design schools, thereby enriching student learning and providing valuable resources for professors. Further work on the platform will involve detailed development of the system architecture, system implementation and formulation of the collaboration protocols.

\section{REFERENCES}

Anumba C. J., Pan J., Issa R. A \& Mutis I. (2008). “Collaborative Project Information Management in a Semantic Web Environment." Engineering Construction and Architectural Management, 15(1), 78-94.

Anumba C. J. (2004). "Information and Communication Technologies to Facilitate Collaboration in Architecture, Engineering and Construction." Collaborative Design and Learning: Competence Building for Innovation, Bento J., Duarte J. P., Heitor M. V. \& Mitchell W. J. (Eds), Praeger Publishers, Westport, USA, 173-195.
Blaszczyszyn, M. (2002). "Day-to-day Reality of Web-based Collaboration - Tools among European Architect Professionals." Connecting the Real and the Virtual - design e-ducation [20th eCAADe Conference Proceedings] Warsaw, Poland, 110-113.

Burka, L. P. (1993). "A hypertext history of multi-user dimensions." MUD history, http://www.ccs.neu.edu/home/lpb/ mud-history.html $>$ (Dec. 5, 1994).

Cheng, N. Y. and Kvan, T. (2000). "Design collaboration strategies." Timmermans, Harry (Ed.), Fifth Design and Decision Support Systems in Architecture and Urban Planning - Part one: Architecture Proceedings, Nijkerk, Netherlands, 45-49.

Fisher, J. W., and Struik, J. H. A. (1974) Guide to design criteria for bolted and riveted joints, Wiley, New York.

Fruchter R., Saxena K., Breidenthal M. and Demian P., Collaborative Design Exploration in an Interactive Workspace, Artificial Intelligence for Engineering Design, Analysis and Manufacturing, Vol. 21, No. 3, 2007, pp 279-293.

International Conference of Building Officials (ICBO). (1997). Uniform building code, Whittier, Calif.

Kurtz, M. (1991). "Section 7: Transformation of space in computer graphics." Handbook of applied mathematics for engineers and scientists, McGraw-Hill, New York, 7.1-7.42.

McCormick, R. (2004). "Collabortaion: The Challenge of ICT." International Journal of Technology and Design Education, 14, 159-176.

Kalay Y. E. (2004). "Virtual Learning Environments." ITcon Special Issue ICT Supported Learning in Architecture and Civil Engineering, 9, 195-207.

Karakaya, F. and Şenyapıl1, B. (2008). "Rehearsal of Professional Practice: Impacts of Web-based Collaborative Learning on the Future Encounter of Different Disciplines." International Journal of Technology and Design Education, 18 (1), 101-117.

Koohang A. and Durante A. (2003). "Learners Perceptions toward the Web-based Distance Learning Activities/ Assignments Portion of an Undergraduate Hybrid Instructional Model." Journal of Information Technology Education, 2, 105-113.

Nguyen T. T. and Bodi J. M. (2007). "Online Teaching: The Development of a Hybrid Course in Higher Education." in K. Kumpulainen (ed.) Educational Technology Opportunities and Challenges, Oulu University Press, Finland, 24-29.

Pentilla, H. (2006). "Describing the Changes in Architectural Information Technology to Understand Design Complexity and Free-Form Architectural Expression." ITCon, 11, 395-408.

Rosen, D. E. and Purinton, E. (2004). "Website Design: Viewing the Web as a Cognitive Landscape." Journal of Business Research 57 (7), 787-794.

Sak, S. (2013). Cyberspace as a Collective Locus for Urban Collective Memory (unpublished master's thesis) Senyapili, B. (supervisor), Bilkent University.

Shelbourn M. A., Bouchlaghem N. M., Anumba C. J. \& Carrillo P. M. (2006). "Developing a DecisionMaking Framework for Effective Collaborative Working." Proceedings of the 13th ISPE International Conference on Concurrent Engineering: Research and Applications, 18th-22nd September, Antibes, France, 103-110.

Sigle J., Key J., Critcher, A. and Prince J. (2005). “Tools for Hybrid Instruction- Classroom and Distance Instruction in Synchronous and Asynchronous Modes." Information Systems Education Journal, 3 (14), 3-7. 
Simoff S. J. and Maher M.L. (1997). "Design Education via Web-based Environments." in Proceedings of 4th ASCE Congress of Computing in Civil Engineering, ASCE, New York, NY, 418-425.

Oxman R. (2007). "Digital Architecture as a Challenge for Design Pedagogy: Theory, Knowledge, Models and Medium.” Design Studies, 29(2), 99-120.

Wang, J. (2012) Challenging ICT Applications in Architecture, Engineering, and Industrial Design Education, IGI Global, Hersey.
Winkler, R. (2002). Keywords and Definitions around "Collaboration", SAP Design Guild, Edition 5, available online http://www.sapdesignguild.org/editions/edition5/ what_is.asp $>$ (Dec. 2013).

Yeung C., Cheung L., Yen J. and Cheng C. (1998). "Virtual Classroom for Architecture." T Sasada, S. Yamaguchi, M. Morozumi, A. Kaga and R. Homma (eds.) CAADRIA '98: Proceedings of the 3rd Conference on Computer Aided Architectural Design Research in Asia, Osaka University, Osaka, 93-102. 